\title{
Acoustic Solitary Waves and Sagdeev Potential Triple Roots
}

\author{
M. A. Hellberg ${ }^{1 *}$, T. K. Baluku ${ }^{\dagger}$ and F. Verheest** \\ ${ }^{*}$ University of KwaZulu-Natal, Durban, 4001, South Africa \\ ${ }^{1}$ hellberg@ukzn.ac.za \\ $\dagger$ University of KwaZulu-Natal, Durban, South Africa, and \\ Mbarara University of Science and Technology, Mbarara, Uganda \\ ${ }^{* *}$ Universiteit Gent, B-9000 Gent, Belgium, and \\ University of KwaZulu-Natal, Durban, South Africa
}

\begin{abstract}
Both KdV theory and the standard pseudopotential theory require that solitons and double layers be explicitly super-acoustic, with the pseudopotential $\psi(\phi, M)$ having a maximum at the origin. Recent studies of a variety of different three-component plasmas have shown that they may support finite amplitude solitons at the true acoustic speed of the plasma configuration, $M_{s}$. These are associated with triple roots of the Sagdeev potential, and the usual soliton condition is replaced by $\psi^{\prime \prime}(0, M) \leq 0$. Sagdeev potentials for speeds marginally greater than $M_{s}$ then represent solitons of both polarities, one whose amplitude vanishes at $M_{s}$ (KdV-like), while the other is necessarily finite at $M_{s}$ ('nonKdV-like'). Such coexistence regions have been observed to be linked to a critical plasma compositional parameter value for which $\psi^{\prime \prime \prime}\left(0, M_{s}\right)=0$.
\end{abstract}

Keywords: Solitons, Sagdeev potential

PACS: $52.35 . \mathrm{Sb}$

\section{INTRODUCTION}

There are many records of satellite observations of nonlinear solitary electrostatic structures, for instance, Refs. [1, 2, 3]. These may typically show a 'spiky' or a bipolar electric field structure. The former is characteristic of a 'kink' in potential, which is associated with adjacent layers of opposite charge, hence called a double layer (DL), while the latter represents a solitary wave (often loosely called a soliton) in the electrostatic potential. Weakly nonlinear solitary waves and double layers are often studied using Reductive Perturbation Theory (RPT), which is equivalent to an expansion in the electrostatic potential $\phi$. This yields an evolution equation for $\phi$, the Korteweg-de Vries $(\mathrm{KdV})$ equation, of the form [4]

$$
\frac{d \phi}{d \tau}+A \phi \frac{d \phi}{d \xi}+B \frac{d^{3} \phi}{d \xi^{3}}=0
$$

The coefficients $A$ and $B$ are dependent on the equilibrium parameter values of the plasma model. Assuming a solitary structure that propagates unchanged at a normalized speed $M$, one obtains the usual solution of the $\mathrm{KdV}$ equation, which may be expressed 
in the laboratory frame as [5]

$$
\phi(x, t)=\frac{3 \delta M}{A} \operatorname{sech}^{2}\left[\left(\frac{\delta M}{4 B}\right)^{1 / 2}(x-M t)\right],
$$

where $\delta M=M-M_{S}>0$ is the increment of the normalized speed of the propagating nonlinear structure over the acoustic speed, $M_{s}$. From this one can deduce some of the characteristics of the KdV model, viz.,

(i) The amplitude of a KdV soliton is proportional to $\left(M-M_{S}\right)$.

(ii) It vanishes for $M=M_{s}$, and hence $\mathrm{KdV}$ solitons are explicitly super-acoustic.

(iii) The polarity of the KdV soliton has the same sign as $A$, i.e., for a specific choice of parameter values the expression can only yield one solution.

Hence $\mathrm{KdV}$ theory cannot explain situations where the plasma can support solitons of either polarity, as have been found by numerous authors, for instance, Refs. [6, 7, 8].

As opposed to the weakly nonlinear KdV model, the Sagdeev (pseudo)potential treatment [9] is fully nonlinear: it is sometimes called an arbitrary amplitude study. One sets up a multifluid description in the frame co-moving with the nonlinear electrostatic structure. Integration of Poisson's equation then yields [9]

$$
\frac{1}{2}\left(\frac{d \phi}{d \xi}\right)^{2}+\psi(\phi, M)=0 .
$$

This equation is akin to an energy integral for a particle of unit mass in a potential well,

$$
\frac{1}{2}\left(\frac{d x}{d t}\right)^{2}+V(x)=0 .
$$

We thus see that the Sagdeev (pseudo)potential $\psi(\phi, M)$, the electrostatic potential $\phi$ and the displacement $\xi$ are mathematically equivalent to the potential $V$, the displacement $x$, and time $t$ in the analogous mechanical equation. Following on from that, one can show that $\psi$ must form a potential well for the resultant function to represent a soliton or double layer. We should point out that the Sagdeev approach does not allow one to confirm the characteristic behaviour of a soliton, viz., that in a soliton collision, the two structures pass through each other without being affected. Nevertheless, we shall, as is commonly done in the literature, use the term 'soliton' loosely for the solitary waves that are found. We also note that, like the $\mathrm{KdV}$ coefficients, $\psi(\phi, M)$ is a function also of the compositional parameters of the plasma.

For the existence of solitons, some conditions have to be satisfied by the pseudopotential, viz.,

(i) $\psi(0, M)=0$, for undisturbed conditions for $\xi \rightarrow \pm \infty$, and

(ii) $\psi^{\prime}(0, M)=0$ represents overall charge neutrality of the equilibrium. Here primes represent derivatives of $\psi(\phi, M)$ with respect to $\phi$.

In the standard pseudopotential approach, the final solitary wave requirement is

(iii) $\psi^{\prime \prime}(0, M)<0$. Thus $\phi=0$ is a local maximum of $\psi(\phi, M)$, i.e., the origin $\phi=0$ is an unstable point. One notes that the acoustic speed $M=M_{S}$ can be shown to be given by $\psi^{\prime \prime}\left(0, M_{s}\right)=0$. Hence in the standard Sagdeev approach, too, the nonlinear solitary 
structures are truly super-acoustic, i.e., $M>M_{S}$, and no solitons exist at the (linear) acoustic speed itself.

\section{RECENT RESULTS}

Contrary to common beliefs, recent studies of a number of physically very different three-component plasmas have yielded finite solitons and double layers at the actual acoustic speed of the plasma configuration $[10,11,12,13]$.
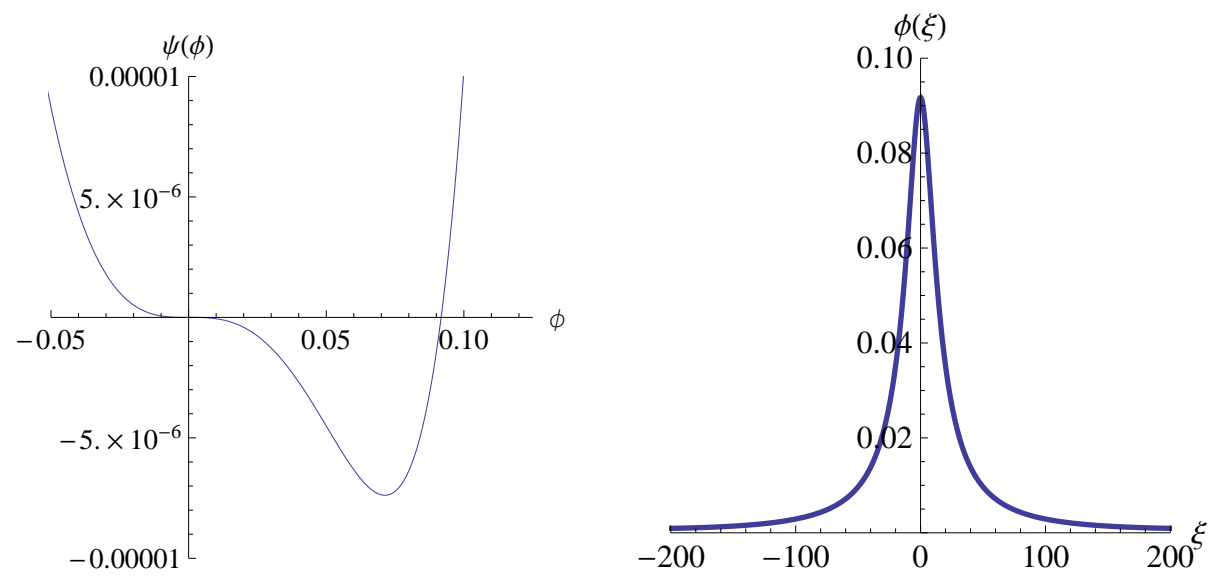

FIGURE 1. Pseudopotential plot (Left Panel) and associated soliton potential profile (Right Panel) for dust ion acoustic solitons at the acoustic speed, $M=M_{s} \simeq 0.835$, for $f=N_{e 0} / N_{i 0}=0.5$, with $\psi^{\prime \prime \prime}\left(0, M_{S}\right)<0$ (positive 'nonKdV-like' soliton). Figures reprinted from Ref. [11] with permission.

As they do not satisfy the $\mathrm{KdV}$ requirement, $\phi \rightarrow 0$ for $M \rightarrow M_{s}$, we call these structures 'nonKdV-like' solitons and double layers [11, 13], where we are again using the term soliton loosely. These nonKdV-like solitons are associated with 'one-sided' pseudopotential wells at $M=M_{s}$, where $\psi^{\prime \prime}(0, M)=0$. The origin is then a triple root of the Sagdeev potential at $M=M_{s}$, and the third derivative $\psi^{\prime \prime \prime}\left(0, M_{s}\right)$ provides the required convexity, i.e., for $\psi^{\prime \prime \prime}\left(0, M_{s}\right)<(>) 0$, the origin $(\phi=0)$ is 'unstable' for $\phi>(<) 0$ at $M=M_{s}$. Although such one-sided wells have in practice been found to yield solitons propagating at $M=M_{s}$, we should point out that that does not necessarily follow, as in principle the $\psi$ curve could break down for some physical reason before yielding a root.

In this section we shall use illustrative examples from Ref. [11], in which we have studied dust ion acoustic (DIA) solitons in a plasma composed of mobile dust (generally with negative charge), adiabatic fluid ions and kappa-distributed electrons. The kappa velocity or energy distribution is frequently encountered in space physics and is used to fit distributions that have a non-Maxwellian tail, with spectral index $\kappa \rightarrow \infty$ yielding a Maxwellian [14, 15]. An example of a one-sided pseudopotential well at the acoustic speed is shown in Fig. 1, together with the associated finite electrostatic soliton.

Arising from the existence of finite solitons at the acoustic speed, it follows that the 'Sagdeev soliton requirement' should be written as $\psi^{\prime \prime}(0, M) \leq 0$, rather than in the usual form, $\psi^{\prime \prime}(0, M)<0$. 

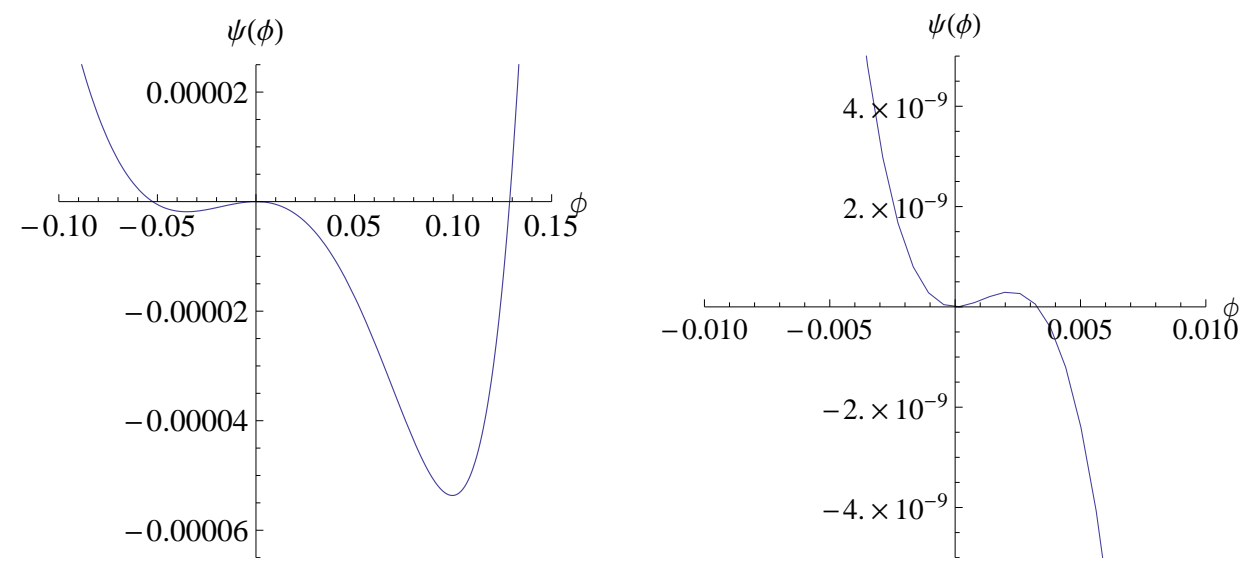

FIGURE 2. Pseudopotential plots for DIA solitons at $f=0.5$, with (Left Panel) $M=M_{s}+0.0023$ $\left(\psi^{\prime \prime}(0, M)<0\right.$; negative 'KdV-like' soliton added), and (Right Panel) $M=M_{s}-0.0001$ ( $\psi$ has no potential well when $M<M_{s}$ and $\left.\psi^{\prime \prime}(0, M)>0\right)$. Figures reprinted from Ref. [11] with permission.

From Fig. 1 we note that $\psi^{\prime \prime \prime}\left(0, M_{S}\right)<0$, and that the associated nonKdV-like soliton has positive polarity. In general, one can show that, for $M>M_{s}$, KdV-like solitons have the same sign as $\psi^{\prime \prime \prime}\left(0, M_{S}\right)$, and if they exist, the nonKdV-like solitons have the opposite sign to $\psi^{\prime \prime \prime}\left(0, M_{s}\right)$ : see Fig. 2 (left panel). This seems to be characteristic for solitons of both polarities occurring in a given plasma configuration, in what is sometimes called the 'coexistence' region of parameter space. Indeed, one can prove that, if a finite amplitude soliton is found to exist at the acoustic speed, $M_{s}$, for a given plasma configuration, then Sagdeev potentials for $M$ marginally above $M_{s}$ will yield solitons of both polarities. A detailed proof will be given elsewhere. Fig. 2 (right panel) also shows that, as expected, there is no soliton for $M$ marginally below $M_{s}$, confirming that our results are not numerical artifacts.

We turn next to consideration of a special (critical) composition of the plasma. Choosing a fixed value of other plasma variables, the typical plasma composition parameter that we vary is most often $f$, the fractional density of one of the two species having a common charge sign. In the case of Ref. [11], we have, for a start, kept $\kappa$ and other physical variables fixed, and varied the fractional equilibrium electron density $f=N_{e 0} / N_{i 0}$. In our studies we have also on occasion varied other important plasma variables. One can find one or more critical values $f_{c}$ by solving the equation, $\psi^{\prime \prime \prime}\left(0, M_{s}\right)=0$. This is equivalent to a choice of parameter values and Mach number that at $\phi=0$ yields a quadruple root

$$
\psi(0, M)=0 ; \psi^{\prime}(0, M)=0 ; \psi^{\prime \prime}(0, M)=0 ; \psi^{\prime \prime \prime}(0, M)=0 .
$$

Using the $\mathrm{KdV}$ approach, one can show that $A=0$ yields a critical plasma composition at which the soliton changes sign (see Eq. [2]), that coincides with this value $f=f_{c}$. As a result, it follows that the $\mathrm{KdV}$-like solitons found here change polarity at $f_{c}$. In fact, we will show later that, if they exist, the nonKdV-like solitons also change sign at the critical compositional parameter value $f_{c}$.

The left panel of Fig. 3 shows that nonKdV-like positive solitons are found for $f_{p}<f=0.5<f_{c} \simeq 0.52$ for a fixed spectral index value $\kappa=2$, where $f_{p}$ is the 

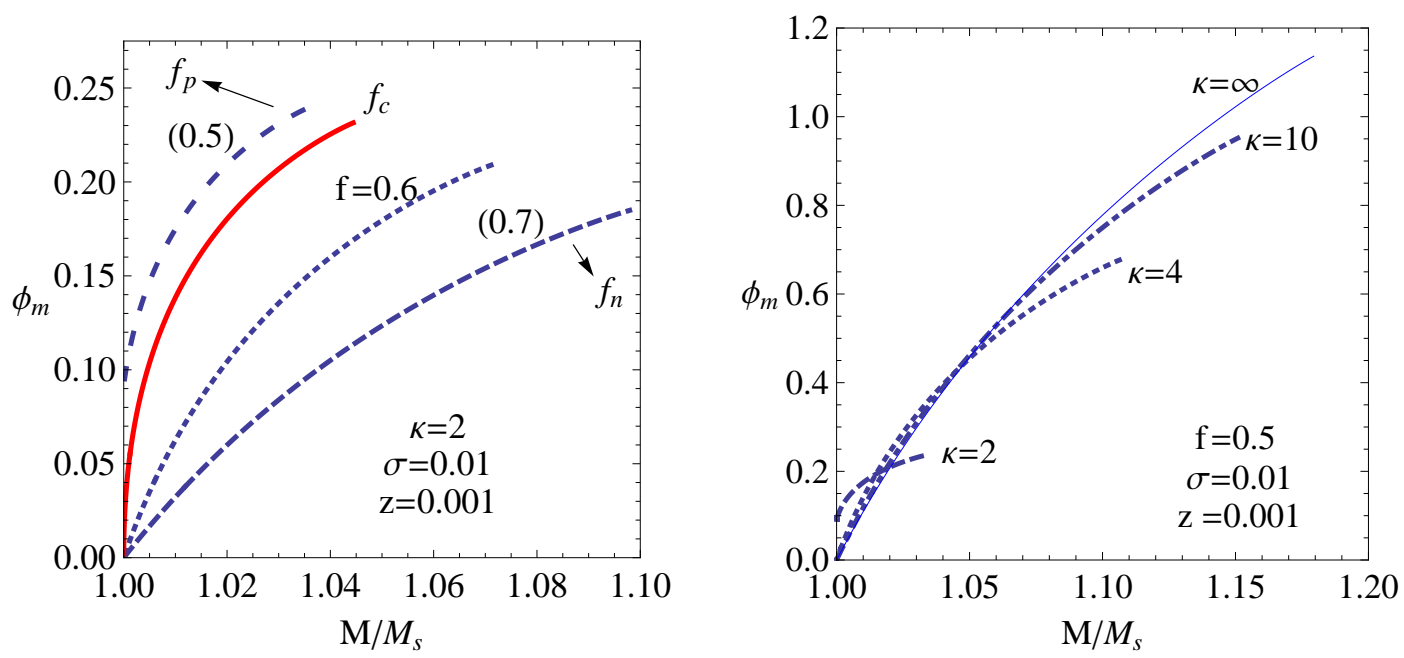

FIGURE 3. Plot of $\phi_{m}$ vs $M / M_{s}$ for positive DIA solitons for (Left Panel) different $f$ in the range $\left(f_{p}, f_{n}\right)$; and (Right Panel) for different $\kappa$. Note the finite positive solitons at $M=M_{s}$ for $f=0.5<f_{c} \simeq$ 0.52 (Left), and for $\kappa=2$, but not for $\kappa \geq 4$ (Right). Figures reprinted from Ref. [11] with permission.

lower cutoff in $f$ for the existence of positive solitons. In the figure $f_{n}$ represents the upper $f$-limit for negative solitons. In the right panel of Fig. 3 we choose a fixed value of electron density, $f=0.5$, and consider different values of $\kappa$. In this case, one sees that positive solitons have finite amplitude at the acoustic speed for $\kappa=2$, but not for $\kappa \geq 4$. So clearly, for $f=0.5$, there is a critical value of $\kappa$, such that $2<\kappa_{c}<4$. Thus we see that for fixed $\kappa$, the critical compositional variable is $f$, while for fixed $f$, the existence of nonKdV-like solitons depends on the value of $\kappa$. The parameter space is multi-dimensional, and the critical parameter values are embedded in such a hyperspace.

In the cases that we have studied, we have found that if there is a region of coexistence, then one of the critical values $f_{c}$ lies either within the coexistence region, or at its edge. Whether only one of these occurs, or both, appears to depend on the details of the model, the assumptions, and the choices of parameter values.

Although obviously the amplitude of a nonKdV-like soliton exceeds that of the KdVlike soliton close to $M=M_{S}$, that does not necessarily hold for larger $M$, where the $\mathrm{KdV}$-like soliton amplitude may be larger than that of the nonKdV-like structure.

\section{OVERVIEW OF PHYSICAL MODELS}

In studying solitary structures propagating in multi-species plasmas, one may define plasma components as being subsonic, i.e., the structure speed is less than the species' thermal speed, $V<v_{t h, j}$ (the species has a 'high' thermal speed), or supersonic $(V>$ $v_{t h, j}$ ), i.e., having a 'low' thermal speed [16]. For such nonlinear structures to exist, one needs to have at least one subsonic and at least one supersonic species [16]. Threecomponent models can be classified according to the number of subsonic and supersonic species, and where there are two species, their relative polarities. We have found finite solitons at the acoustic speed, and associated phenomena discussed above, in a range of 
physically very different three-component systems:

1. Two subsonic species having the same sign.

We have considered ion acoustic (IA) solitons in plasmas with double Boltzmann electrons, and cold ions [13]. This study has been extended to IA solitons in a plasma with double kappa electrons, and adiabatic ions [17].

2. Two subsonic species having opposite signs.

We have studied dust acoustic (DA) solitons in a plasma with Boltzmann electrons and hotter 'Cairns' nonthermal ions, together with adiabatic negative dust [10]. We have also considered DA solitons in a model with subsonic electrons and ions both having a Cairns nonthermal distribution, and cold negative dust [12].

Finally, we have very recently found the same behaviour in modified IA solitons observed in an electron-positron-ion plasma in which the electrons are Cairns-distributed, the positrons Boltzmann-distributed, and the ions cold [17]. We note that the Cairns distribution [8] was introduced as a model to simulate observed nonthermal distributions in space, and importantly, was shown to support solitons of either polarity.

3. Two supersonic species having opposite signs.

Finite dust ion acoustic solitons at $M=M_{S}$ were found in a plasma with adiabatic ions and cold negative dust, together with kappa electrons [11]. As a special case, $\kappa=\infty$, this study included Boltzmann electrons.

But interestingly, if one switched to positive dust, and thus considered two supersonic species having the same sign, it was found that $\psi^{\prime \prime \prime}\left(0, M_{S}\right)>0$ throughout the range of $f:$ there was no coexistence region, and there were no finite solitons at $M=M_{s}$ [11]. Thus having two supersonic species of the same sign does not in general support these newly-reported structures. We cannot yet say whether they can exist for such plasmas at all.

4. In addition to the above three-component plasma studies, we have very recently also found the same phenomenon for the original two-component Cairns model, but assuming cold ions [18]. In this case the only plasma parameter which can yield a critical value is the nonthermal parameter $\beta$, which thus leads to a critical $\beta_{c}$ in place of the $f_{c}$ considered above [18]. We point out that in Ref. [8] this nonthermal distribution was shown to support a coexistence region. However, the presence of finite solitons at the acoustic speed, the associated triple roots of the pseudopotential, and the 'nonKdV' nature of one of the two polarities were first discussed in Ref. [18].

\section{A DETAILED EXAMPLE}

As an example we shall discuss IA solitary structures in a plasma with cold ions and two Boltzmann electron components, which is discussed in more detail in Ref. [13]. This is a well-known model, having been studied earlier by numerous authors, for example, in chronological order, Refs. [19, 20, 6, 7]. The normalized Sagdeev potential can be written as [13]

$$
\begin{aligned}
\psi(\phi, M)= & \frac{f}{\alpha_{c}}\left[1-\exp \left(\alpha_{c} \phi\right)\right]+\frac{1-f}{\alpha_{h}}\left[1-\exp \left(\alpha_{h} \phi\right)\right] \\
& +M^{2}\left[1-\left(1-2 \phi / M^{2}\right)^{1 / 2}\right] .
\end{aligned}
$$



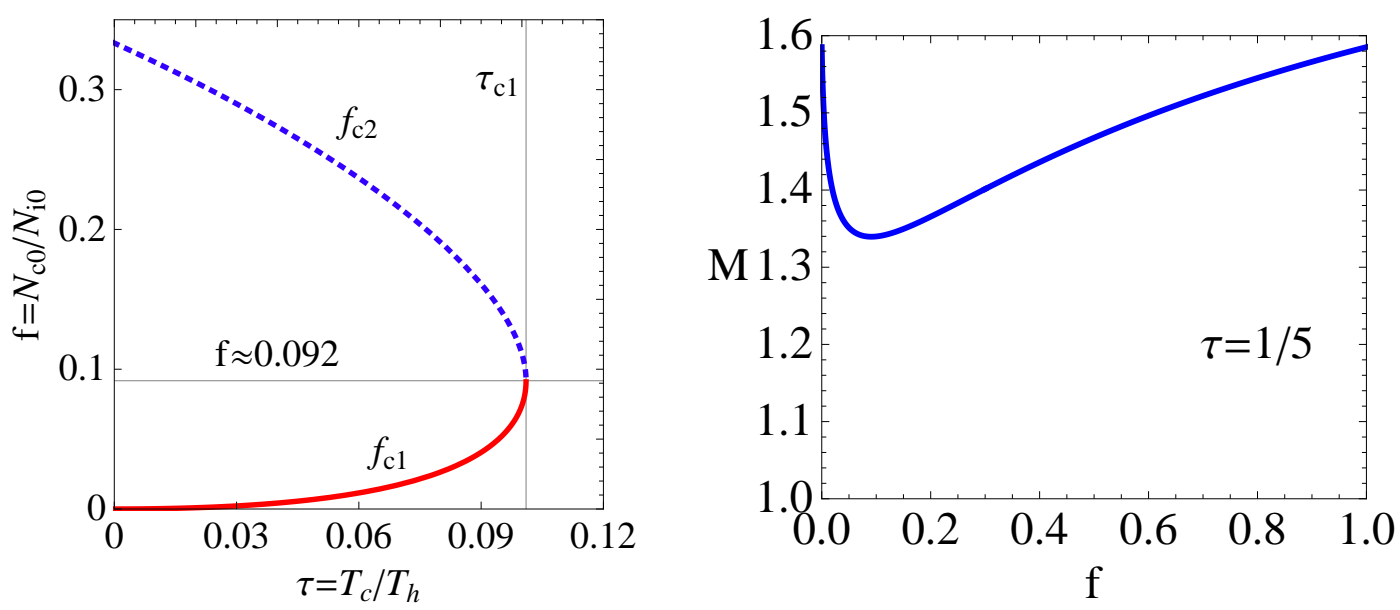

FIGURE 4. Left Panel: Variation of the critical density fraction, $f_{c}$, with $\tau$, along the curve, $\psi^{\prime \prime \prime}\left(0, M_{s}\right)=0$. Inside the curve, $\psi^{\prime \prime \prime}\left(0, M_{S}\right)<0$, and one has negative KdV-like solitons, while outside the curve, $\psi^{\prime \prime \prime}\left(0, M_{S}\right)>0$ and KdV-like solitons are positive. Right Panel: Existence domain of ion acoustic solitons for $\tau=0.2>\tau_{c 1}$. Positive potential solitons exist for all $f$ from 0 to 1 , and are bounded by the ion density compression limit. Figures reprinted from Ref. [13] with permission.

Here the temperature ratio is $\tau=T_{c} / T_{h}$, the cool electron density fraction is $f=N_{c 0} / N_{i 0}$, one may define an effective temperature $T_{\text {eff }}=T_{c} /(f+[1-f] \tau)$, the normalized potential is expressed w.r.t. $K_{B} T_{\text {eff }} / e$, and the Mach number $M$ is relative to the true ion acoustic speed $C_{S}=\left(K_{B} T_{\text {eff }} / m_{i}\right)^{1 / 2}$, that is, $M=M_{S}$ is equivalent to $M=1$. The critical value of the composition parameter, $f_{c}$, may be found from

$$
\psi^{\prime \prime \prime}\left(0, M_{s}\right)=3-\frac{f+\tau^{2}(1-f)}{(\tau[1-f]+f)^{2}}=0 .
$$

The form of this expression underlines the fact that, as noted earlier, the 'critical value' is not just a point, but is in general multi-dimensional. In this case, it is a curve in the two-dimensional space of $\tau$ and $f$. For fixed $\tau$, two critical values $f_{c 1,2}$ exist if $\tau \leq \tau_{c 1} \simeq 0.10102$. At $\tau=\tau_{c 1}$, roots $f_{c 1}$ and $f_{c 2}$ coalesce into a single root $f_{c} \simeq 0.092$.

All figures that follow are taken from Ref. [13], although captions have been edited. We first show the critical curve in the $[\tau, f]$ plane in the left panel of Fig. 4 [13]. This figure is not new: Ref. [19] reported one of the $f_{c}$ when investigating rarefactive shocks in laser plasmas, and a figure equivalent to Fig. 4 was found by Ref. [6], using a different approach, viz., through consideration of what they call regions of anomalous and normal propagation. We draw attention to the fact that inside the critical curve, $\psi^{\prime \prime \prime}\left(0, M_{S}\right)<0$, and one has negative $\mathrm{KdV}$-like solitons, while outside the curve (and thus for all $\tau>\tau_{c 1}$ ), $\psi^{\prime \prime \prime}\left(0, M_{S}\right)>0$, and $\mathrm{KdV}$-like solitons are positive.

The right panel shows the existence domain of positive solitons for $\tau>\tau_{c 1}$ [13]. It stretches over the full range of electron densities from $f=0$ to 1 , and for a Mach number $M$ ranging from 1 to an $f$-dependent upper limit that arises from the density compression limit of the ions, $M=M_{\ell i}$. For such a value of $\tau$, no negative solitons are found, nor double layers of either polarity. This result is well-known $[6,7]$. 

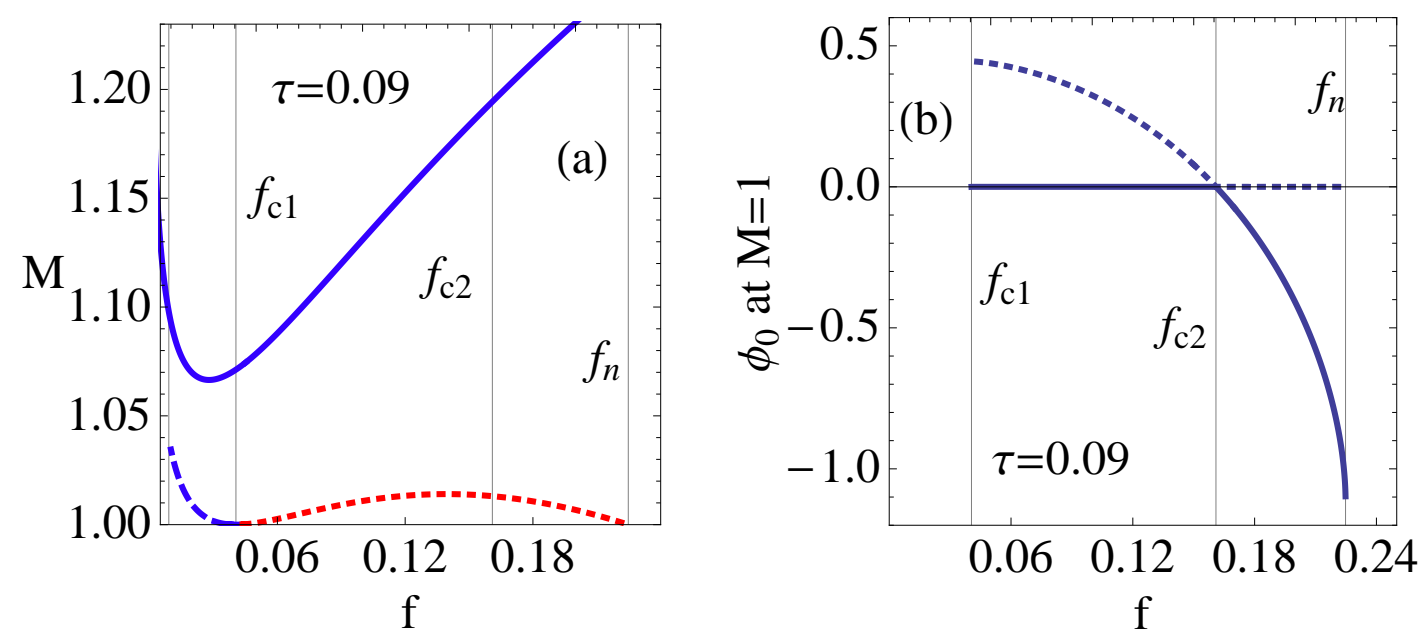

FIGURE 5. Left Panel: Existence domain of solitons and double layers for $\tau=0.09<\tau_{c 1}$. The upper continuous curve is the ion compression limit, that is lower than in Fig. 4 (right panel). The lower left dashed curve represents positive DLs; the negative structure limit is the lower right dotted curve. The 'coexistence region' lies between $f_{c 1}$ and $f_{n}$. Right Panel: Soliton amplitudes at the acoustic speed $M=M_{s}=1$, covering the range of $f$ for the coexistence region. Here $f_{c 1} \simeq 0.041, f_{c 2} \simeq 0.161$ and $f_{n} \simeq 0.225$. Both $\mathrm{KdV}$-like and nonKdV-like solitons switch sign at $f_{c 2}$, while the KdV-like solitons also switch sign at $f_{c 1}$. Note the large amplitude DL at $M=M_{s}$ for $f=f_{n}$. Figures reprinted from Ref. [13] with permission.

We next turn to $\tau=0.09<\tau_{c 1}$. From Fig. 4 (left panel) we see that there are now two distinct critical $f$ values, $f_{c 1}$ and $f_{c 2}$. This case is illustrated in Fig. 5, from Ref. [13]. As expected, both solitons and double layers (DLs) have a lower limit of $M=1$. In the left panel of Fig. 5, the upper continuous curve again shows the upper limit of positive solitons, due to ion compression, at $M_{\ell i}$. We note, however, that it is lower than was the case for $\tau>\tau_{c 1}$ in Fig. 4 (right). The lower right (dotted) curve is an upper limit for negative solitons associated with the forming of negative DLs, at a Mach number that we shall call $M_{d l}$. In this range of $f$, negative solitons will occur for $1<M<M_{d l}$.

The negative solitons and DLs occur in the range $f_{c 1}<f<f_{n}$, both the upper limit $f_{n}$ and the lower limit being values of $f$ for which $M_{d l}=1$, i.e., a DL occurs at the acoustic speed, and no negative solitons can be found for those values of $f$. On the other hand, positive solitons are possible from $f=0$ up to an upper limit $f_{p}$ which is greater than $f_{n}$. Thus over the range $\left[f_{c 1}, f_{n}\right]$, solitons of both polarities are supported, i.e., this range is a region of parameter space in which the two polarities can occur - loosely called a 'coexistence region'. So the lower critical value $f_{c 1}$ lies at the edge of the coexistence region. It separates the dotted curve $M_{d l}$ for negative DLs from the lower left dashed curve, that represents positive DLs. On the other hand, it is seen that the other critical value of $f, f_{c 2}$, lies squarely within the coexistence range, and we shall show below that it plays a different role in the existence domain.

The right panel of Fig. 5 provides interesting information on the amplitudes of solitons and DLs at the acoustic speed $M=M_{s}=1$ in a region of $f$ that covers the coexistence range $\left[f_{c 1}, f_{n}\right]$. The dotted sections show the amplitude at the acoustic speed of those structures that are associated with positive solitons at $M_{s}$ (for $f<f_{c 2}$ ) or (above $f_{c 2}$ ) 
for slightly increased values of $M, M>M_{s}$. On the other hand, the continuous curves are the equivalent negative structures. It is seen that for $f_{c 1}<f<f_{c 2}$, negative solitons have zero amplitude at the acoustic speed, and are thus KdV-like, while positive solitons have finite amplitude for $M=M_{s}$, and are nonKdV-like. For $f_{c 2}<f<f_{n}$ the situation is reversed, with positive solitons being KdV-like, and negative solitons being finite at the acoustic speed. Thus $f_{c 2}$ is seen to separate these two subregions within the coexistence region.

If we consider the value $\phi_{0}=0$, we see that the $\mathrm{KdV}$-like structures that satisfy this requirement at $M=M_{s}$ change sign at the upper critical point, $f=f_{c 2}$. The same obviously applies to the curved nonKdV-like sections. Hence another view on the role of $f_{c 2}$ is to say that the $\mathrm{KdV}$-like and nonKdV-like structures change sign at the critical point, as was commented on in an earlier section. Finally, a third description of this observation is that as one increases $f$, the positive solitons switch from being of non-KdV form to being $\mathrm{KdV}$-like, while the negative solitons switch from $\mathrm{KdV}$-like to nonKdV-like at $f_{c 2}$. We have pointed out earlier that at $f=f_{n}$, there are no negative solitons, but there is a DL with $M=M_{d l}=1$. From this figure one can see that this DL at $f_{n}$ has a very large amplitude that is of order 1 in normalized form.

Finally, we need to comment briefly on an aspect of the left panel of Fig. 5 that is important, even though it is not immediately relevant to Sagdeev triple roots. We point out that there appears to be a contradiction, viz., there are two curves that should normally represent upper limits for the positive solitons. On the one hand, the ion density limit $M_{\ell i}$ is an obvious upper limit, as it relates to the limit of the range in $M$ having finite real ion density. On the other hand, it has commonly been observed, for instance in [7] and elsewhere, that DLs (plotted as the lower left dashed curve) represent a limit on a sequence of solitons. So the question arises, what occurs for Mach numbers lying between these two apparent 'upper limits'?

The question is discussed in detail in Ref. [13]. In brief, the oft-quoted DL-limit [7] applies to Sagdeev potentials of relatively straightforward shape, as in [7], but if the pseudopotential has sufficient structure to yield enough local maxima and minima, it need not represent an upper limit for $M$. Instead, as $M / M_{S}$ is increased the soliton amplitude increases continuously with $M$ (as is well-known from other studies, e.g., $[21,11])$, but at $M=M_{d l}$ the curve shows a discontinuous jump to a higher amplitude value, before the soliton range is eventually cut off at $M=M_{\ell i}$, the ion density limit.

\section{CONCLUSIONS}

Using the arbitrary amplitude, Sagdeev potential approach, we have studied conditions for the existence of solitary waves and double layers, and some of their characteristics, in a number of physically very different three-component plasmas. In all cases studied we have found some common threads:

(i) Contrary to common beliefs, finite solitons and double layers may be found at the true acoustic speed for the plasma configuration, $M=M_{S}$, defined by $\psi^{\prime \prime}\left(0, M_{S}\right)=0$. We term these structures nonKdV-like, as they do not satisfy the $\mathrm{KdV}$ and $\mathrm{mKdV}$ requirements of vanishing at the acoustic speed.

(ii) The origin $(\phi=0)$ is then a triple root of the Sagdeev (pseudo)potential. 
(iii) Hence the usual Sagdeev soliton existence condition $\psi^{\prime \prime}(0, M)<0$ is more generally $\psi^{\prime \prime}(0, M) \leq 0$.

(iv) $\mathrm{KdV}$-like solitons have the same sign as $\psi^{\prime \prime \prime}\left(0, M_{S}\right)$, while, in a coexistence region of parameter space, in which solitons of both polarities are supported, nonKdV-like structures have the opposite sign.

(v) At a critical plasma composition, given by $\psi^{\prime \prime \prime}\left(0, M_{S}\right)=0$, the KdV-like and, if they exist, nonKdV-like solitons switch polarity. Put differently, solitons of a given polarity switch between $\mathrm{KdV}$-like and nonKdV-line form there.

(vi) We have been able to prove analytically that if $\psi\left(0, M_{S}\right)^{\prime \prime \prime} \neq 0$, and in addition a nonKdV-like soliton exists at $M=M_{s}$, then for $M>M_{s}$ but close to $M_{s}$, a KdV-like solitary structure will also exist, and thus both polarities are supported.

\section{ACKNOWLEDGMENTS}

Valuable discussions with Ioannis Kourakis and Nareshpal Singh Saini are gratefully acknowledged. This research was supported in part by the National Research Foundation (NRF) of South Africa. T.K.B. also thanks NASSP for its financial support.

\section{REFERENCES}

1. R. Boström, G. Gustafsson, B. Holback, G. Holmgren, and H. Koskinen, Phys. Rev. Lett. 61, 82 (1988).

2. R. E. Ergun, C. W. Carlson, J. P. McFadden, F. S. Mozer, G. T. Delory, W. Peria, C. C. Chaston, M. Temerin, I. Roth, L. Muschietti, R. Elphic, R. Strangeway, R. Pfaff, C. A. Cattell, D. Klumpar, E. Shelley, W. Peterson, E. Moebius, and L. Kistler, Geophys. Rev. Lett. 25, 2041 (1998).

3. R. E. Ergun, L. Andersson, J. Tao, V. Angelopoulos, J. Bonnell, J. P. McFadden, D. E. Larson, S. Eriksson, T. Johansson, C. M. Cully, D. N. Newman, M. V. Goldman, A. Roux, O. Lecontel, K.-H. Glassmeier, and W. Baumjohann, Phys. Rev. Lett. 102, 155002 (2009).

4. P. K. Shukla and A. A. Mamun, Introduction to Dusty Plasma Physics (Institute of Physics, Bristol, U.K., 2002), p. 197.

5. R. L. Mace, S. Baboolal, R. Bharuthram, and M. A. Hellberg, J. Plasma Phys. 45, 323 (1991).

6. K. Nishihara and M. Tajiri, J. Phys. Soc. Japan 50, 4047 (1981).

7. S. Baboolal, R. Bharuthram, and M. A. Hellberg, J. Plasma Phys. 44, 1 (1990).

8. R. A. Cairns, A. A. Mamun, R. Bingham, R. Boström, R. O. Dendy, C. M. C. Nairn, and P. K. Shukla, Geophys. Res. Lett. 22, 2709 (1995).

9. R. Z. Sagdeev, in: Reviews of Plasma Physics 4 (Ed. M.A. Leontovich, Consultants Bureau, New York, 1966), p. 23.

10. F. Verheest and M. A. Hellberg, Phys. Plasmas 17, 023701 (2010).

11. T. K. Baluku, M. A. Hellberg, I. Kourakis, and N. S. Saini, Phys. Plasmas 17, 053702 (2010). Figures 1-3 reprinted with permission. Copyright 2010, American Institute of Physics.

12. F. Verheest, Phys. Plasmas 17, 062302 (2010).

13. T. K. Baluku, M. A. Hellberg, and F. Verheest, Europhys. Lett. 91, 15001 (2010). Figures 4-5 reprinted with permission.

14. V. M. Vasyliunas, J. Geophys. Res. 73, 2839 (1968).

15. M. A. Hellberg, R. L. Mace, T. K. Baluku, I. Kourakis, and N. S. Saini, Phys. Plasmas 16, 072113 (2009).

16. F. Verheest, T. Cattaert, G. S. Lakhina, and S.V. Singh, J. Plasma Phys. 70, 237 (2004).

17. T. K. Baluku, Studies of Linear and Nonlinear Waves in Space Plasmas, Ph.D. Thesis, University of KwaZulu-Natal, South Africa (Submitted August, 2010). 
18. F. Verheest and M. A. Hellberg, Compressive and rarefactive solitary waves in nonthermal twocomponent plasmas, Phys. Plasmas 17 (Accepted, September, 2010).

19. B. Bezzerides, D. W. Forslund, and E. L. Lindman, Phys. Fluids 21, 2179 (1978).

20. B. Buti, Phys. Lett. A 76, 251 (1980).

21. N. S. Saini, I. Kourakis, and M. A. Hellberg, Phys. Plasmas 16, 062903 (2009). 